\title{
Spinal epidural hematoma related to an epidural catheter in a cardiac surgery patient -A case report-
}

Jiyoun Bang, Joung Uk Kim, Yu Mi Lee, Junghwa Joh, Eun-Hye An, Jae-young Lee, Ji Yeon Kim, and In-cheol Choi

Department of Anesthesiology and Pain Medicine, Asan Medical Center, University of Ulsan College of Medicine, Seoul, Korea

The addition of thoracic epidural anesthesia to general anesthesia during cardiac surgery may have a beneficial effect on clinical outcome. However, epidural catheter insertion in a patient anticoagulated with heparin may increase the risk of epidural hematoma. We report a case of epidural hematoma in a 55-year-old male patient who had a thoracic epidural placed under general anesthesia preceding uneventful mitral valve replacement and tricuspid valve annular plasty. During the immediate postoperative period and first postoperative day, prothrombin time (PT) and activate partial thromboplastin time (aPTT) were mildly prolonged. On the first postoperative day, he complained of motor weakness of the lower limbs and back pain. An immediate MRI of the spine was performed and it revealed an epidural hematoma at the T5-6 level. Rapid surgical decompression resulted in a recovery of his neurological abnormalities to near normal levels. Management and preventing strategies of epidural hematoma are discussed. (Korean J Anesthesiol 2011; 61: 524-527)

Key Words: Analgesia, Epidural, Hematoma, Postoperative complications, Spinal.

A combination of general anesthesia with thoracic epidural anesthesia may have a beneficial effect on clinical outcomes after cardiac surgery [1]. There is concern, however, about its use in patients with perioperative anticoagulation, because of the risk of bleeding, which could cause serious adverse events like epidural hematoma and neurologic injury [2,3]. We describe here a spinal epidural hematoma related to epidural analgesia in an adult patient who underwent cardiac surgery.
On the day of surgery, an epidural catheter was preoperatively placed for postoperative analgesia. After 16 hours from the end of operation, the patient complained of flaccid paraplegia with sensory deficits and back pain. Surgical evacuation performed 6 hours after the symptoms first developed. Due to rapid treatment, patient's neurological condition was markedly improved.

Received: April 4, 2011. Revised: 1st, July 26, 2011; 2nd, August 25, 2011; 3rd, September 15, 2011. Accepted: September 15, 2011.

Corresponding author: Yu Mi Lee, M.D., Ph.D., Department of Anaesthesiology and Pain Medicine, Asan Medical Center, University of Ulsan College of Medicine, 388-1, Pungnap-2dong, Songpa-gu, Seoul 138-736, Korea. Tel: 82-2-3010-3870, Fax: 82-2-3010-6790, E-mail: ylee@amc.seoul.kr (c) This is an open-access article distributed under the terms of the Creative Commons Attribution Non-Commercial License (http:// creativecommons.org/licenses/by-nc/3.0/), which permits unrestricted non-commercial use, distribution, and reproduction in any medium, provided the original work is properly cited. 


\section{Case Report}

A 55-year-old male patient weighing $65 \mathrm{~kg}$ was scheduled for an elective mitral valve replacement and tricuspid annular plasty for treatment of severe mitral and tricuspid valve regurgitation. His significant past medical history included atrial fibrillation and diabetes mellitus. His current medications included warfarin, glimepiride, torasemide, verapamil, and isosorbide dinitrate.

Warfarin was discontinued 7 days before elective surgery. Routine preoperative laboratory tests revealed a normal clotting screen with an activated partial thromboplastin time (aPTT) of $37.8 \mathrm{~s}$, a prothrombin time (PT) of 1.08 INR and a normal platelet count of $168,000 / \mathrm{mm}^{3}$.

On the day of surgery, an epidural catheter was placed at the T7-8 level after the induction of general anesthesia for postoperative analgesia. After the patient was positioned in the right lateral decubitus position by an assistant, the epidural space was identified using a paramedian approach and a loss of resistance to air by a $16 \mathrm{G}$ Tuhoy needle at T7-8 level. An epidural catheter was threaded $3 \mathrm{~cm}$ into the epidural space and secured over the patient's back. These procedures were atraumatic, with no bloody tap or blood in the catheter. The patient was anesthetized for surgery with total intravenous anesthesia using midazolam and fentanyl. Intravenous unfractionated heparin 19,500 U (300 U/kg) started on for cardiopulmonary pump 130 minutes after epidural catheterization. The activated clotting time (ACT) was monitored and maintained adequate level to keep the anticoagulated state during the operation. Upon completion of the valve replacement and intravenous protamine (293 mg) was administered to reverse the effect of heparin. A continuous epidural infusion of preservative free sufentanil $500 \mu \mathrm{g}$ and $0.18 \%$ ropivacaine $250 \mathrm{ml}$ was used for postoperative analgesia with baseline infusion rate $1 \mathrm{ml} / \mathrm{hr}$, bolus $2 \mathrm{ml}$ and lockout time 15 minutes.

On the examination 1 hour after surgery while conscious in the intensive care unit, the patient showed clear mental status and neurologically intact movement. His immediate postoperative PT (INR) and aPTT were 1.52 and $42.2 \mathrm{~s}$, respectively, and his platelet count was $77,000 / \mathrm{mm}^{3}$. After 16 hours from the end of cardiac surgery, the patient complained of flaccid paraplegia with sensory deficit and back pain. There was oozing at the insertion site of the epidural catheter. At this time, his aPTT was $53.3 \mathrm{~s}$, and his PT was 1.30 INR. An initial examination revealed posterior midthoracic tenderness to palpation, flaccid paraplegia, loss of deep tendon reflexes in the lower extremities and anus, hyperesthesia in bilateral T5 -7 dermatomes, and anesthesia of both lower extremities. Immediately, he was taken an emergency magnetic resonance imaging (MRI) of the spine, which revealed an epidural hematoma at the T5-6 level (Fig. 1). The patient underwent an emergency $\mathrm{T} 5-\mathrm{T} 7$ laminectomy and the spinal epidural hematoma was completely removed within 6 hours after the symptoms first developed. Seven days after laminectomy, the patient had almost fully regained his motor and sensory functions, but he still experienced dysuria and right foot drop. Six months after laminectomy, his motor and sensory functions had fully recovered, while dysuria was slight.

\section{Discussion}

A combination of general anesthesia with thoracic epidural anesthesia (TEA) may have an additional beneficial effect
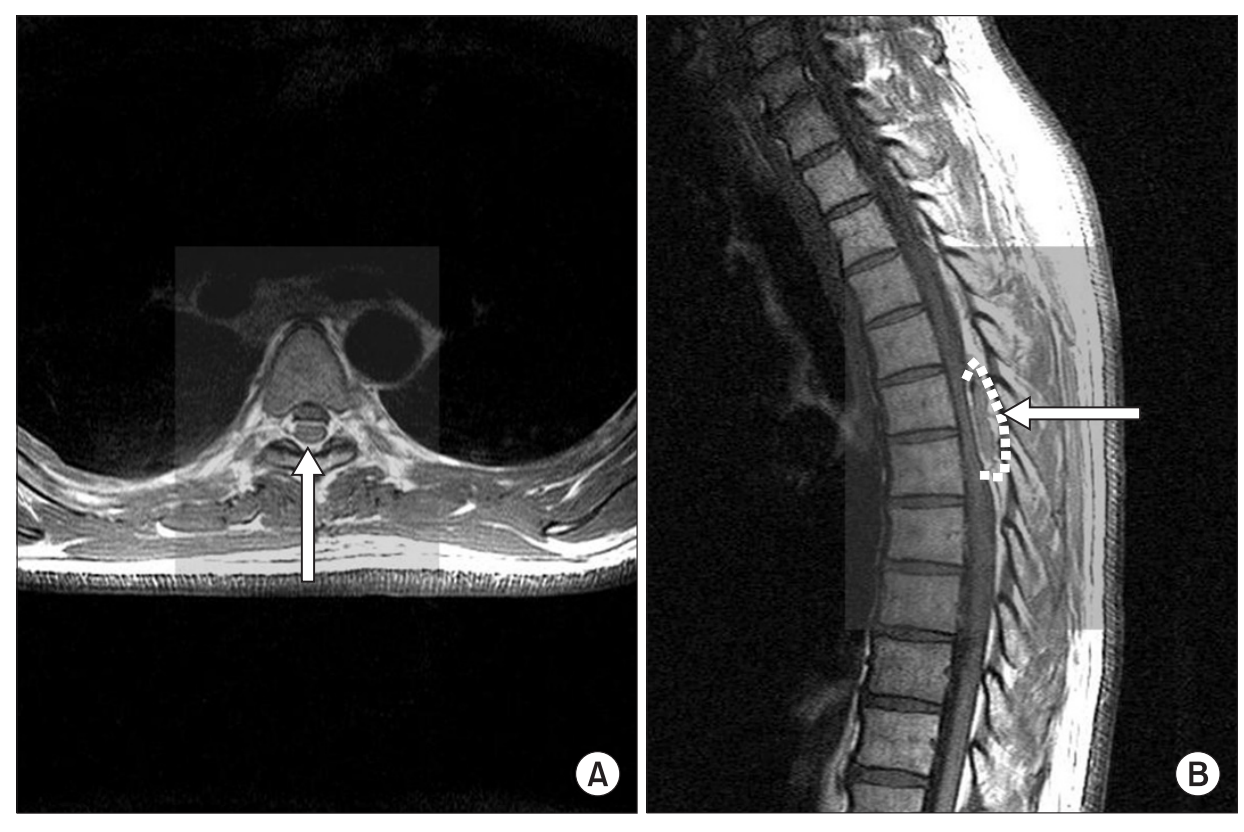

Fig. 1. The MRI of the spinal epidural hematoma. Axial T2 weighted images at the T6 levels demonstrate an epidural hematoma returning low signal (white arrow in A). The spinal epidural hematoma compresses the epidural sac along the T5-7 level (white arrow in B). There is no definite signal change at T 5-7 level. 
outcome on cardiac surgery, compared with general anesthesia. TEA may enhance coronary perfusion, improve myocardial oxygen balance, and reduce the incidence of tachyarrhythmias and perioperative myocardial ischemia through sympatholysis [1]. The excellent analgesia that is associated with TEA facilitates early tracheal extubation and may prevent respiratory complications [4]. However, concerns have arisen about the use of epidural anesthesia in patients receiving perioperative anticoagulation therapy, due to an enhanced risk of bleeding, which could cause serious adverse events such as epidural hematoma and neurologic injury $[2,5]$. In spite of these concerns, several published reports have shown that TEA is safe in adult patients undergoing surgery requiring intraoperative anticoagulation [6]. For the safe use of the neuraxial technique in patients receiving anticoagulants, the following precautions have been recommended [7]: (1) This technique should not be used in patients with any form of known coagulopathy; (2) surgery should be delayed 24 hours in the event of a traumatic tap; (3) the time from instrumentation to systemic heparinization should exceed 60 minutes; (4) the amount of heparin and its reversal should be tightly controlled, using the smallest amount of heparin for the shortest duration compatible with therapeutic objectives; (5) epidural catheters should be removed when normal coagulation is restored, and (6) patients should be closely monitored postoperatively for signs and symptoms of hematoma formation.

However, despite adhering to these guidelines, the first case of an epidural hematoma related to insertion of an epidural catheter before cardiac surgery has been reported by Rosen et al. in 2004 [8]. The epidural hematoma developed after the use of heparin and a thrombolytic agent in the postoperative period, and was likely due to postoperative anticoagulation [8]. In our patient, it is uncertain when the epidural hematoma developed especially. We kept to the guidelines strictly and the thoracic epidural catheter insertion was atraumatic that is there was no blood in the epidural needle or catheter. The epidural catheter should be inserted to the patient under conscious state to monitor the neurologic complication, but it would be significantly painful process to the patient. Therefore, we inserted the catheter after induction of general anesthesia to minimize the patient's discomfort. Heparin was administered 130 minutes after the placement of the epidural catheter. Our patient experienced postoperative coagulation abnormalities, including prolonged PT and thrombocytopenia. However, the abnormalities were not treated to keep anticoagulated state to prevent thromboembolic event caused by enhanced thrombin generation after cardiopulmonary bypass surgery [9]. While he seemed to be neurologically intact during the immediate postoperative period, neurologic abnormalities were observed the next morning on the first operative day, after 16 hours from the end of operation. We have deduced some possible mechanisms for delayed appearance of symptom.

First, the epidural venous plexus might have been injured during the insertion of epidural needle and catheter. Epidural bleeding, likely because of injury to venous tissues, occurs slowly and require time to accumulate to a clinically significant level. The patient might have shown neurologically intact movement during the immediate postoperative period because the accumulated amount of hemorrhage was insufficient to produce the compressive effect. The continuous hemorrhage due to lack of blood clot formation by postoperative coagulopathy begins to accumulate in the epidural cavity and compress the spinal cord. Second, the epidural hematoma might have occurred due to postoperative coagulopathy. It sounds reasonable because the patient seemed to have intact neurologic function during the immediate postoperative period. Moreover, if the epidural bleeding had been occurred during the instrumentation, there were oozing though the epidural catheter or around the insertion site because of full heparinization during cardiopulmonary bypass. Lastly, although it has been reported that epidural hematoma could occur spontaneously [10], we do not believe that this was the case in our patient.

This patient's first symptom was motor weakness of the lower limbs and back pain. New onset muscle weakness and back pain are the most frequent related symptoms of epidural hematoma, observed in $46 \%$ and $38 \%$ of patients, respectively. Other initial signs and symptoms of epidural hematoma include sensory deficit and urinary retention, observed in $14 \%$ and $8 \%$ of patients, respectively [2]. Although spontaneous recovery of spinal epidural hematoma has been reported, immediate surgical decompression is the treatment of choice to prevent further deterioration of neurologic functions $[11,12]$. Clinical factors influencing neurologic prognosis include the severity of preoperative neurologic deficits, speed of onset of clinical signs, location of the hematoma, age, duration of spinal cord compression, and the time interval between onset of clinical signs and surgical evacuation [11,12]. Decompression within eight hours of the initial development of neurologic signs and symptoms has been associated with good neurologic outcomes [2]. Fortunately, in our patient, the neurologic abnormalities and the presence of an epidural hematoma were identified early. The time from detection of neurologic symptoms to decompressive laminectomy was 6 hours, making possible his neurologic recovery to near normal levels.

Based on estimates of the incidence of epidural hematomas in the absence of anticoagulation ( $1: 150,000-1: 220,000)$ [7], a sample of nearly 200,000 patients would be required for $80 \%$ power to detect a 10 -fold increase in the incidence of this complication [13]. Therefore, since studies of this magni- 
tude have not been performed, it remains unclear whether epidural catheterization in patients undergoing cardiac surgery is safe. Due to insufficient data, it was unclear whether preoperative placement of an epidural catheter followed by full heparinization one to three hours later would decrease this risk [8]. Some authors have failed to demonstrate a clinically relevant benefit of thoracic epidural analgesia on the frequency of major complications after elective cardiac surgery, compares with fast-track cardiac anesthesia without epidural anesthesia [3]. Given the potentially devastating complications of an epidural hematoma after insertion of an epidural catheter, it is questionable whether this procedure should be applied routinely in cardiac surgical patients who required full heparinization.

In summary, we have described the occurrence of a postoperative epidural hematoma in a patient whose coagulation system was altered during and after surgery with heparin. The patient also had postoperative thrombocytopenia, further contributing to derangement of his coagulation system. These factors contributed to the development of an epidural hematoma on postoperative day 1 . However, early detection of neurologic abnormalities and rapid diagnosis of epidural hematoma led to early cord decompression and good neurologic prognosis. Judicious selection of candidates and cautiously attempting catheterization one day before surgery are necessary, as is the monitoring of these patients for neurologic function and symptoms of epidural hematoma.

\section{References}

1. Nygård E, Kofoed KF, Freiberg J, Holm S, Aldershvile J, Eliasen K, et al. Effects of high thoracic epidural analgesia on myocardial blood flow in patients with ischemic heart disease. Circulation 2005; 111 : 2165-70.

2. Vandermeulen EP, Van Aken H, Vermylen J. Anticoagulants and spinal-epidural anesthesia. Anesth Analg 1994; 79: 1165-77.

3. Svircevic V, Nierich AP, Moons KG, Diephuis JC, Ennema JJ, Brandon Bravo Bruinsma GJ, et al. Thoracic epidural anesthesia for cardiac surgery: a randomized trial. Anesthesiology 2011; 114: 26270.

4. Ballantyne JC, Carr DB, deFerranti S, Suarez T, Lau J, Chalmers TC, et al. The comparative effects of postoperative analgesic therapies on pulmonary outcome: cumulative meta-analyses of randomized, controlled trials. Anesth Analg 1998; 86: 598-612.

5. Svircevic V, van Dijk D, Nierich AP, Passier MP, Kalkman CJ, van der Heijden GJ, et al. Meta-analysis of thoracic epidural anesthesia versus general anesthesia for cardiac surgery. Anesthesiology 2011; 114: 271-82.

6. Chaney MA. Intrathecal and epidural anesthesia and analgesia for cardiac surgery. Anesth Analg 2006; 102: 45-64.

7. Horlocker TT, Wedel DJ, Rowlingson JC, Enneking FK, Kopp SL, Benzon HT, et al. Regional anesthesia in the patient receiving antithrombotic or thrombolytic therapy: American Society of Regional Anesthesia and Pain Medicine Evidence-Based Guidelines (Third Edition). Reg Anesth Pain Med 2010; 35: 64-101.

8. Rosen DA, Hawkinberry DW 2nd, Rosen KR, Gustafson RA, Hogg JP, Broadman LM. An epidural hematoma in an adolescent patient after cardiac surgery. Anesth Analg 2004; 98: 966-9.

9. Lison S, Dietrich W, Braun S, Boehm J, Schuster T, Englhard A, et al. Enhanced thrombin generation after cardiopulmonary bypass surgery. Anesth Analg 2011; 112: 37-45.

10. Ahn SS, Song YJ. Clinical experience and management of cervicothoracic epidural hematoma. J Korean Neurosurg Soc 2010; 47: 3814.

11. Foo D, Rossier AB. Preoperative neorological status in predicting surgical outcome of spinal epidural hematomas. Surg Neurol 1981; 15: 389-401.

12. Lawton MT, Porter RW, Heiserman JE, Jacobowitz R, Sonntag VK, Dickman CA. Surgical management of spinal epidural hematoma: relationship between surgical timing and neurological outcome. J Neurosurg 1995; 83: 1-7.

13. Urmey WF, Rowlingson J. Do antiplatelet agents contribute to the development of perioperative spinal hematoma? Reg Anesth Pain Med 1998; 23(6 Suppl 2): 146-51. 